\title{
Molecular and Cellular Basis of Deficiency of the b Subunit for Factor XIII Secondary to a Cys430-Phe Mutation in the Seventh Sushi Domain
}

\author{
Teruto Hashiguchi and Akitada Ichinose \\ Department of Molecular Patho-Biochemistry, Yamagata University School of Medicine, Yamagata, 990-23 Japan
}

\begin{abstract}
We studied the defect responsible for deficiency of the $b$ subunit for factor XIII in the first known case of this condition. The patient is a compound heterozygote of two genetic defects: deletion of $\mathrm{A}-\mathbf{4 1 6 1}$ at the acceptor splice junction of intron $A$, resulting in a loss of the obligatory $\underline{A} G$ splicing sequence; and, replacement of G-11499 by $T$ in exon VIII, resulting in an amino acid substitution of Cys430 by Phe. To determine how the latter mutation impaired $b$ subunit synthesis, recombinant $b$ subunit bearing the mutation was expressed in BHK cells. The mutant as well as wild-type $b$ subunit was synthesized by the cells. However, the apparent molecular weight of the mutant was slightly higher than those of the wild-type and plasma $b$ subunits under nonreducing conditions, probably because of destruction of a disulfide bond. The mutant $b$ subunit was secreted from the cells much less effectively than the wild type and remained susceptible to endoglycosidase $H$, indicating that it was not transported from the endoplasmic reticulum to the Golgi apparatus where the processing of oligosaccharides occurs. Immunofluorescence study suggested that the mutant protein was retained in the endoplasmic reticulum. These studies demonstrate that a Cys430-Phe mutation does not prevent the de novo synthesis of the $b$ subunit, but alters the conformation of the mutant protein sufficiently to impair its intracellular transport, resulting in its deficiency in this patient. (J. Clin. Invest. 1995. 95:1002-1008.) Key words: bleeding disorder $\bullet$ in vitro mutagenesis • protein conformation • impaired transport $\cdot$ ER retention
\end{abstract}

\section{Introduction}

Coagulation factor XIII is a plasma transglutaminase consisting of two catalytic $a$ and two noncatalytic $b$ subunits. The $a$ subunit is also present as an $a_{2}$ dimer in platelets/megakaryocytes, monocytes/macrophages, skin dendrocytes, placenta, and prostate, whereas the $b$ subunit is synthesized in hepatocytes (for

This paper was presented in part at the 16th Congress of the Japanese Society on Thrombosis and Hemostasis, December 2-3, 1993, Tokyo, Japan.

Address correspondence to Akitada Ichinose, M.D., Ph.D., Department of Molecular Patho-Biochemistry, Yamagata University School of Medicine, Iida-Nishi 2-2-2, Yamagata, 990-23 Japan. Phone: 23633-6829; FAX: 236-24-4534.

Received for publication 15 August 1994 and in revised form 31 October 1994.

J. Clin. Invest.

(C) The American Society for Clinical Investigation, Inc. 0021-9738/95/02/1002/07 \$2.00

Volume 95, March 1995, 1002-1008 reviews see references $1-3$ ). Since the $a$ subunit carries the active site of the enzyme, its deficiency leads directly to defective cross-linking reactions and, as a consequence, to bleeding, abnormal wound healing, and spontaneous abortion in females. Intracranial bleeding is the leading cause of death in factor XIII deficiency. Although many cases of factor XIII deficiency have been reported, most of them are deficiencies of the $a$ subunit. Recently, the first patient with complete deficiency of the $b$ subunit was found in Japan (4). The patient also has a reduced plasma level of the $a$ subunit and manifests a mild bleeding tendency. It is highly likely that the lack of the $b$ subunit resulted in the loss of the $a$ subunit because of its instability. Indeed, an accelerated half-life of therapeutic exogenous $a$ subunit has been observed in this patient.

The amino acid sequence of factor XIII has been established by a combination of gene cloning and amino acid sequence analysis (5-8). The $a$ subunit consists of 731 amino acids, while the $b$ subunit is composed of 641 residues which form ten tandem repeats called Sushi domains or GP-I structures (9). Determination of the genomic sequence for both $a$ and $b$ subunits has made it possible to characterize factor XIII deficiency at the genetic level (10-14). Genomic DNAs obtained from the proband of complete $b$ subunit deficiency (4) and her family members have been examined by Southern blotting analysis and in vitro amplification employing gene-specific primers (14). DNA sequence analysis revealed that an adenosine was deleted in half of the amplified DNAs at the acceptor splice junction of intron A, resulting in a loss of the obligatory AG splicing sequence (see Fig. 3 in reference 14). Furthermore, a guanosine coding for Cys430 ( T $\underline{\text { GC }}$ ) in exon VIII was replaced by a thymidine in half of the amplified DNAs, resulting in an amino acid change to Phe (TTC) and destruction of a disulfide bond in the seventh Sushi domain of the $b$ subunit. Thus, the proband was a genotypic compound heterozygote of two defects. Her daughter and son are genotypic heterozygotes of the second mutant allele. It is not clear, however, how the Cys430-Phe mutation induces the $b$ subunit deficiency.

In the present study, we constructed expression vectors containing cDNAs coding for either wild type (normal) or the Cys430-Phe mutant of the $b$ subunit and transfected the resulting vectors into mammalian cells to elucidate the mechanism of the $b$ subunit deficiency. The expressed $b$ subunits were examined for their cellular biological and biochemical properties. Pulsechase experiments and immunocytochemical analyses demonstrated that the intracellular transport of the mutant from the endoplasmic reticulum to the Golgi apparatus was impaired despite the presence of newly synthesized mutant protein. Thus, the Cys430-Phe mutation was confirmed as the cause of the $b$ subunit deficiency by in vitro mutagenesis studies in baby hamster kidney $(\mathrm{BHK})^{1}$ cells.

1. Abbreviations used in this paper: BHK, baby hamster kidney; endo $H$, endoglycosidase $H$; ER, endoplasmic reticulum; Met, methionine. 


\section{Methods}

Construction of expression vectors for wild-type and mutant $b$ subunits. ZMB3 (15) is an expression vector for mammalian cells and was kindly provided by $C$. Sprecher (Zymogenetics Inc., Seattle, WA). A cDNA coding for the $b$ subunit of human factor XIII in the mammalian expression vector, ZMB3/XIII $b$ (ZMB/XIIIB in reference 12), was released and subcloned into M13mp18 for mutagenesis. An oligonucleotide primer, 5'-AGTACATGGTTCCAAGAAAACAGGTGGGGA-3', was synthesized on an Applied Biosystems synthesizer (Foster City, CA) and used for in vitro mutagenesis of an amino acid residue at 430 from Cys to Phe. Mutagenesis was performed using Oligonucleotide-directed in vitro mutagenesis system version 2.1 (Amersham, Arlington Heights, IL) by the method of Taylor et al. (16). The mutation in the cDNA was confirmed by dideoxy sequencing and the cDNA was then inserted into ZMB3 (Fig. 1). The constructed expression vectors for the wild type and mutant $b$ subunits were designated ZMB3/XIII $b$ and ZMB3/ MXIII $b$, respectively.

Cell culture and transfection. Baby hamster kidney (BHK) cells were grown in Dulbecco's modified Eagle medium (DME) supplemented with $10 \%$ fetal calf serum (FCS), $2 \mathrm{mM}$ L-glutamine, $50 \mu \mathrm{g} /$ $\mathrm{ml}$ penicillin, $50 \mu \mathrm{g} / \mathrm{ml}$ streptomycin, and $100 \mu \mathrm{g} / \mathrm{ml}$ neomycin (GIBCO BRL, Gaithersburg, MD). For transient expression, approximately 3 $\times 10^{6} \mathrm{BHK}$ cells were transfected with $10 \mu \mathrm{g}$ of the expression plasmid, ZMB3/XIII $b$ or ZMB3/MXIII $b$, by the calcium phosphate method. For stable expression, $48 \mathrm{~h}$ after transfection cells were grown in the culture medium containing $5 \mathrm{mg} / \mathrm{ml} \mathrm{G418} \mathrm{(GIBCO} \mathrm{BRL)} \mathrm{as} \mathrm{a} \mathrm{selective} \mathrm{agent.}$ After $\sim 10 \mathrm{~d}, \mathrm{G} 418$-resistant colonies were isolated and propagated individually in the selection medium containing G418. All cell cultures were maintained in a humidified $5 \% \mathrm{CO}_{2}$ incubator at $37^{\circ} \mathrm{C}$.

ELISA and Immunoblotting of culture media and cell lysates. Cells expressing the wild type or mutant $b$ subunit were cultured in $100-\mathrm{mm}$ dishes (Falcon, Oxnard, CA). After washing with phosphate buffered saline (PBS), the cells were cultured further in $6 \mathrm{ml}$ of culture medium without FCS (serum-free medium). The culture media were harvested after $24 \mathrm{~h}$, and the cells were washed with PBS and lysed in $3 \mathrm{ml}$ of a detergent solution containing $10 \mathrm{mM}$ Tris- $\mathrm{HCl}, \mathrm{pH} 7.5,0.15 \mathrm{M} \mathrm{NaCl}$, $5 \mathrm{mM}$ EDTA, $1 \%$ sodium deoxycholate, $1 \%$ Triton $\mathrm{X}-100$, and $0.1 \%$ SDS.

Concentrations of the wild-type and mutant $b$ subunits in culture media and cell extracts were measured by ELISA, using a rabbit polyclonal antibody against human $b$ subunit (Calbiochem Corp., La Jolla, CA) as the primary antibody, and a peroxidase-conjugated goat antirabbit IgG antibody (Bio Rad Laboratories, Richmond, CA) as the secondary antibody. ELISAs were developed with 3, 3', 5, 5' $5^{\prime}$-tetramethylbenzidine as the colorimetric substrate (Bio Rad), followed by $0.1 \%$ $\mathrm{H}_{2} \mathrm{O}_{2}$.

For Western blotting, three volumes of culture media or cell lysates were mixed with one volume of $4 \times$ sample buffer $(125 \mathrm{mM}$ Tris- $\mathrm{HCl}$, $\mathrm{pH} 6.8,4 \%$ SDS, $50 \%$ glycerol, and $1 \mathrm{mg} / \mathrm{ml}$ bromophenol blue) and boiled for $5 \mathrm{~min}$; then $50 \mu \mathrm{l}$ of aliquots were electrophoresed on $8 \%$ polyacrylamide gels under reducing and nonreducing conditions. Samples were also analyzed by nondenatured polyacrylamide gel electrophoresis without SDS as described by Lorand et al. (17). The separated proteins were transferred onto $0.2 \mu \mathrm{m}$ Polyvinylidene fluoride (PVDF) membranes (Atto Corp., Japan) using a semi-dry electroblotter. The membrane was then blocked at room temperature for $1 \mathrm{~h}$ using a Western buffer containing $50 \mathrm{mM}$ Tris- $\mathrm{HCl}, \mathrm{pH} 7.4,5 \mathrm{mM}$ EDTA, $0.05 \%$ NP$40,0.15 \mathrm{M} \mathrm{NaCl}$, and $0.25 \%$ gelatin, and incubated at room temperature for $1 \mathrm{~h}$ with the rabbit anti- $b$ subunit antiserum at a dilution of 1:1,000 in the Western buffer. After washing the membrane with TBST $(0.02$ $\mathrm{M}$ Tris-HCl. $\mathrm{pH} 7.4,0.15 \mathrm{M} \mathrm{NaCl}$, and $0.05 \%$ Tween-20), a goat antirabbit IgG antibody conjugated with alkaline phosphatase at a 1:1,000 dilution in the Western buffer was added for $1 \mathrm{~h}$ at room temperature. After four washes with TBST, bound antibody was detected by adding nitroblue tetrazolium and 5-bromo-4-chloro-3-indolyl phosphate (Bio $\mathrm{Rad}$ ) for $\mathbf{3 0} \mathrm{min}$ at room temperature.

Pulse-chase experiments and endoglycosidase $H$ treatment. For transient expression, $4 \times 10^{5}$ BHK cells in six-well $(37 \mathrm{~mm})$ plates were transfected with $5 \mu \mathrm{g}$ of ZMB3/XIII $b$ or ZMB3/MXIII $b$ as described above. At $48 \mathrm{~h}$ after transfection, the cells were washed and incubated in methionine-free DME containing 5\% dialyzed FCS for 1 $\mathrm{h}$ at $37^{\circ} \mathrm{C}$. The cells were then labeled with $80 \mu \mathrm{Ci} /$ well of $\left[{ }^{35} \mathrm{~S}\right]-$ methionine (Met) in Met-free DME with 5\% dialyzed FCS for 15 min (pulse), and further incubated in serum-free DME supplemented with unlabeled Met (chase). The chase was terminated at various time intervals, culture media and cell lysates were harvested and stored at $-80^{\circ} \mathrm{C}$ immediately. This study was repeated by using COS-7 (a monkey kidney cell line) cells. For stable expression, all conditions were exactly the same as those for transient expression except that BHK cells which stably produced the wild type or mutant $b$ subunit and $25 \mu \mathrm{Ci} /$ well of $\left[{ }^{35} \mathrm{~S}\right]$ Met were used.

Immunoprecipitation of the $b$ subunit was carried out by incubation of the supernatant of samples with the anti- $b$ subunit antiserum. After $1 \mathrm{~h}$ of incubation at $4^{\circ} \mathrm{C}$ with shaking, a 50\% suspension of protein $\mathrm{A}-$ Sepharose was added to the samples and incubated for $1 \mathrm{~h}$ at $4^{\circ} \mathrm{C}$ with shaking. The immunoprecipitates were washed five times with washing buffer ( $20 \mathrm{mM}$ Tris- $\mathrm{HCl}$, pH 7.5, 0.1\% SDS, $0.1 \mathrm{M} \mathrm{NaCl}, 5 \mathrm{mM}$ EDTA, $0.5 \%$ NP-40) to remove nonspecifically adsorbed proteins. Bound antigen was eluted from the Sepharose beads by heating at $100^{\circ} \mathrm{C}$ for $5 \mathrm{~min}$ and then subjected to electrophoresis on 10\% SDS-polyacrylamide gels, followed by treatment with an enhancer (Amplify; Amersham). The gels were dried and exposed at $-80^{\circ} \mathrm{C}$ to Kodak XAR autoradiography films.

To evaluate the form of oligosaccharide side chains of the wild-type and mutant $b$ subunits, samples were treated with endo- $\beta$ - $N$-acetylglucosaminidase $\mathrm{H}$ (endo $\mathrm{H}$; Boehringer Mannheim Biochemica), which cleaves immature high-mannose type side chains. After being washed, the immunoprecipitates were heated at $100^{\circ} \mathrm{C}$ for $2 \mathrm{~min}$, and endo $\mathrm{H}$ was added to a final concentration of $100 \mathrm{mU} / \mathrm{ml}$. The reaction mixture was incubated at $37^{\circ} \mathrm{C}$ for $16 \mathrm{~h}(18)$. Undigested samples (control) were treated identically without the addition of endo $\mathrm{H}$. Electrophoresis of each sample was performed as described above.

Immunocytochemistry. BHK cell cultures on coverslips contained in $60-\mathrm{mm}$ plates were washed with PBS, fixed with $4 \%$ paraformaldehyde at $4^{\circ} \mathrm{C}$ overnight, and then permealized in $0.1 \%$ Triton X-100 for $15 \mathrm{~min}$ at room temperature. The cells were washed with PBS and incubated with $2 \%$ FCS in PBS for $2 \mathrm{~h}$, then reacted with the antiserum against the $b$ subunit at a dilution of $1: 1,000$ in PBS at $4^{\circ} \mathrm{C}$ overnight. The cells were washed with PBS and followed by incubation for another $2 \mathrm{~h}$ at room temperature with a goat anti-rabbit IgG conjugated with fluorescein-5-isothiocyanate (Organon Teknika Corp., Durham, NC) at a dilution of 1:1,000 in PBS containing 2\% FCS. After four washes with PBS, the coverslips were mounted on microscopic slides and observed under an epifluorescence microscope (Olympus BH-2, Japan).

\section{Results}

Expression of recombinant b subunit for factor XIII in BHK cells. To verify that the Cys430-Phe substitution actually interferes with $b$ subunit synthesis, we introduced the mutation into the cDNA sequence of the wild-type $b$ subunit by site-directed mutagenesis and expressed the mutant protein in BHK cells. It was found by ELISA in preliminary experiments that both the wild-type and mutant $b$ subunits were produced at extremely low levels by transient expression, and the recombinant $b$ subunit was not clearly revealed by Western blotting (data not shown). Therefore, three clones each of the wild type or mutant were selected at random and cultured separately in the culture medium containing G418 for stable expression. Amounts of the $b$ subunit in culture media of the wild-type clones were 55.3, 30.4 , and $18.2 \mu \mathrm{g} / 10^{7}$ cells $/ 24 \mathrm{~h}$ and those of the mutant were $30.0,11.0$, and 2.7. According to yields of the $b$ subunit, these stable clones were designated high-, intermediate-, and low- 
Wild Type (Cys-430)

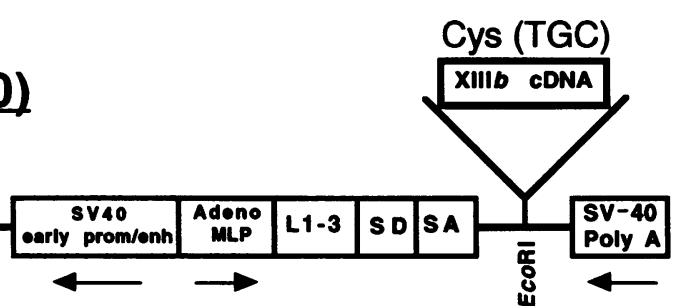

Mutant (Phe-430)

ZMB3

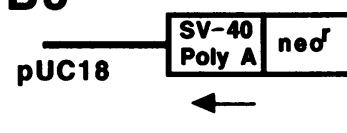

Phe (TIC)

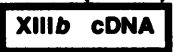

Figure 1. Construction of expression vectors for the $b$ subunits of factor XIII. A cDNA coding for the $b$ subunit of factor XIII was ligated into an EcoRI cloning site downstream of the Adenovirus promoter in an expression vector, ZMB3. $S V-40$ poly $A$, a polyadenylation signal sequence for $\mathrm{SV}-40$; $S V-40$ early prom/enh, early promoter and enhancer of SV-40; Adeno MLP, major late promoter of Adenovirus; $L 1-3$, tripartite leader; $S D$, splice donor; $S A$, splice acceptor from Adenovirus. Arrows indicate directions of transcription.

producers, respectively. The average yield of the mutant was lower than that of the wild-type (14.6 vs. $34.6 \mu \mathrm{g} / 10^{7}$ cells/ $24 \mathrm{~h})$.

Since an average yield of the mutant $b$ subunit in culture media was lower than that of the wild type, a large amount of the mutant could be retained inside cells. In order to test this possibility, amounts of intra- and extra-cellular $b$ subunits were measured by ELISA in the high producers of the wild type and the mutant. Preliminary studies confirmed that the majority of the wild-type $b$ subunit was secreted into the culture medium, while the majority of the mutant was retained inside cells (data not shown), strongly suggesting that secretion of the mutant $b$ subunit from synthesizing cells is impaired as a result of the Cys430-Phe substitution.

The amounts of both the normal and mutant $b$ subunits in culture media did not change when incubated for $4 \mathrm{~d}$ at $37^{\circ} \mathrm{C}$, nor did the bands for the mutant protein decrease during the incubation period (data not shown). Thus, degradation of the $b$ subunit does not seem to be significant in the culture media after its secretion.

Immunoblot of the mutant b subunit containing Cys430Phe. To determine the molecular forms of wild type and mutant $b$ subunits in culture media, two stable clones of high-producers were selected, one each from the wild type and mutant. In contrast to the transient expression system, Western blot analysis could clearly detect both the wild type and mutant recombinant $b$ subunits in the stable expression system (Fig. 2, left panel). The apparent molecular weight of the mutant $b$ subunit, however, was slightly higher than that of the wild type $(\sim 85$ $\mathrm{kD})$. It is very likely that the mutant molecule is extended because one of two disulfide bonds in the seventh Sushi domain is absent. This explanation is consistent with the fact that there was no difference in the apparent molecular weights of the two molecules when the samples were reduced with $\beta$-mercaptoethanol (Fig. 2, right panel).

A band, which may represent a disulfide-bonded covalent dimer of the mutant $b$ subunit, was observed under nonreducing conditions, but disappeared upon reduction. A covalent dimer may have formed during sample preparation and/or after secretion because the mutant $b$ subunit has an unpaired Cys residue, which is absent in the wild-type molecule. It could be that the covalent dimer does not exist inside living cells where protein disulfide isomerase keeps disulfide bonds cleaved. Very faint bands in lower molecular weight regions may represent degradation fragments of the mutant protein. However, both the covalent dimer and lower molecular weight bands were not readily visible in pulse-chase experiments as shown later.

The mutant $b$ subunit as well as the wild type migrated as a dimer when analyzed by non-denatured polyacrylamide gel electrophoresis (data not shown), indicating that the mutant retains the ability to form a noncovalent dimer as shown by Lorand et al. (17).

Pulse-chase experiments in transient and stable expression systems. To evaluate the intracellular transportation of newly synthesized $b$ subunit, clones for the high-producers of the wild type and mutant were used in pulse-chase studies. The highproducer cells were pulse-labeled with $\left[{ }^{35} \mathrm{~S}\right] \mathrm{Met}$ for $15 \mathrm{~min}$ and were chased with culture media containing unlabeled Met for various periods of time before the $b$ subunit was immunoprecipitated using antiserum. Nascent wild-type $b$ subunit seen at $0 \mathrm{~h}$ (Fig. 3) gradually decreased in the cells and appeared in the culture medium at 1 and $2 \mathrm{~h}$. At $4 \mathrm{~h}$, most of the $b$ subunit was

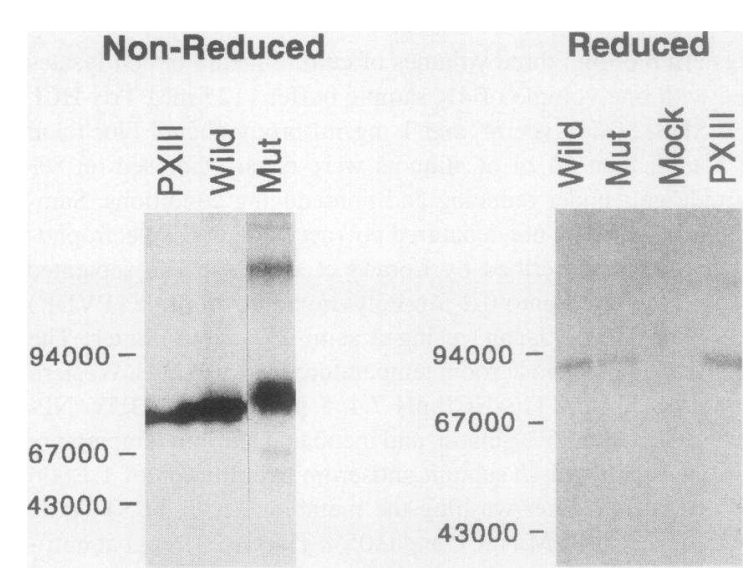

Figure 2. Western blotting of the $b$ subunit. The plasma (PXIII), wildtype (Wild), and mutant (Mut) $b$ subunits in culture media of highproducer clones by stable expression were separated under nonreduced (left) and reduced (right) conditions on 8\% SDS-polyacrylamide gels and detected with the antiserum against $b$ subunit. 


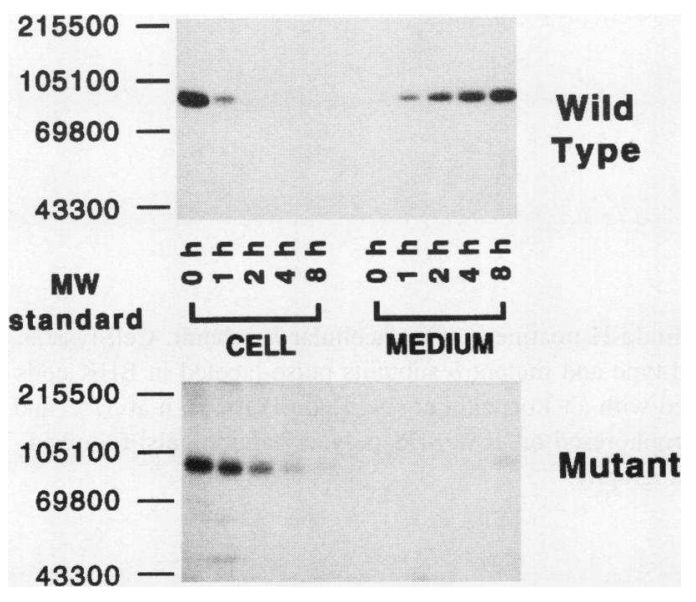

Figure 3. Pulse-chase study by stable expression. Pulse-chase experiments were performed in the stable expression system as described in Methods. Radiolabeled cell lysates (left, intracellular) and culture media (right, extracellular) of high-producer clones were immunoprecipitated with an antiserum against the $b$ subunit, followed by electrophoresis on 10\% SDS-polyacrylamide gels. Top, wild type; bottom, mutant.

found in the culture medium. In contrast, the mutant $b$ subunit in the cells decreased with time but did not appear in the culture medium. Only a small amount of the mutant was detected in the culture medium after $8 \mathrm{~h}$, while all wild type protein was recovered under the same conditions. Similar results were obtained when intermediate producers for the wild type and mutant were used (data not shown). Nearly complete loss of secretion of the Cys430-Phe mutant in BHK cells indicates that it is slowly degraded in an intracellular compartment $(\mathrm{s})$.

Pulse-chase experiments were also performed successfully in the transient expression system. This was possible because of the advantage radiolabeling of proteins has over Western blotting by virtue of high sensitivity. As seen in the stable expression system, the wild type $b$ subunit was secreted quantitatively and the mutant was not released from cells in the transient expression system (Fig. 4). These results strongly suggest that the intracellular transport of the mutant $b$ subunit is impaired.

The pulse-chase experiments used with $\mathrm{COS}-7$ cells provided the same results as described above in BHK cells (data not shown).

Endoglycosidase treatment of intracellular b subunits. Because the wild-type $b$ subunit is secreted from synthesizing cells, while the mutant is not, it could be that the mutant is trapped at some stage (s) in the secretary pathway and does not undergo normal posttranslational processing. To examine Asnlinked oligosaccharide processing of the mutant $b$ subunit, both cell lysates and culture media were incubated with or without endo $\mathrm{H}$, an enzyme with specificity for immature, high-mannose type oligosaccharide side chains.

The wild-type $b$ subunit in the cell lysates was sensitive to endo $\mathrm{H}$ digestion at $0 \mathrm{~h}$, and became resistant after $1 \mathrm{~h}$ (Fig. 5 ). Contrary to the wild type, the intracellular mutant remained susceptible to endo $\mathrm{H}$ during the observed period, indicating that the mutant was retained in a compartment(s) before the medial-Golgi. These results strongly suggest that intracellular transport of the mutant was blocked between the ER and the Golgi apparatus where processing of oligosaccharides occurs.

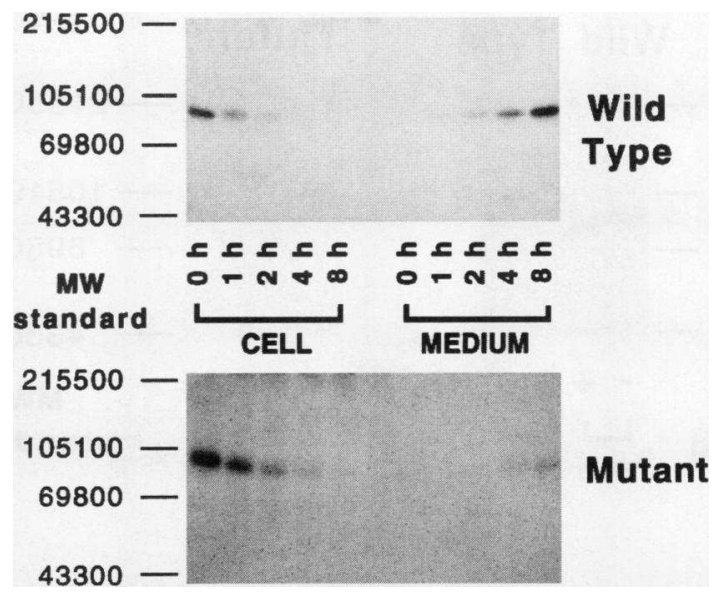

Figure 4. Pulse-chase study by transient expression. Pulse-chase experiments were performed in the transient expression system. Radiolabeled cell lysates (left) and culture media (right) were treated as described in Fig. 3. Top, wild type; bottom, mutant.

Immunocytochemistry. To investigate the subcellular localization of the $b$ subunit, immunocytochemical studies were performed. Fig. 6 shows the immunofluorescence microscopy of three different samples: BHK cells producing the mutant, the wild-type $b$ subunit, and mock cells as a negative control. Bright diffuse fluorescence of a fine punctuate/reticular pattern characteristic of ER labeling was observed when the cells producing the mutant reacted with the anti- $b$ subunit antiserum (Fig. 6 A). Control cells showed no significant fluorescence (Fig. 6 $C)$. This staining pattern is consistent with our hypothesis that the mutant protein remains in the ER (ER retention). In contrast to the cells expressing the mutant, a prominent signal, appearing as a stacked and compact structure near the cell nucleus (Fig. $6 \mathrm{~B}$ ), was observed in addition to a weak diffuse fluorescence pattern inside the cells producing the wild-type $b$ subunit. Although the localization of the $b$ subunit in the cells cannot be absolutely determined by the light microscopic study, these results strongly suggest that the normal protein is present in the Golgi apparatus as well as in the ER. Thus, it is very likely that the wild-type $b$ subunit was transported from the ER to the Golgi apparatus where processing of oligosaccharides occurs, while the mutant was retained in the ER. The impaired intracellular transport of the mutant $b$ subunit can account for its deficiency in this case because the newly synthesized mutant $b$ subunit never reaches circulation.

\section{Discussion}

The five examples of factor XIII deficiency in which the mutation is known (10-13) all result from genetic defects of the $a$ subunit. However, an inherited quantitative abnormality of the $b$ subunit also results in factor XIII deficiency, which is characterized by mild bleeding symptoms (4). In the previous report (14), we determined two genetic mutations in the first patient who has been diagnosed as having complete $b$ subunit deficiency. One of the two mutations, Cys430(TGC)-Phe(TTC) is the only missense mutation to be described to date that causes the $b$ subunit deficiency, the mechanism(s) for which is unclear. In order to determine whether the Cys430-Phe mutation does indeed give rise to the observed phenotype, constructs bearing 


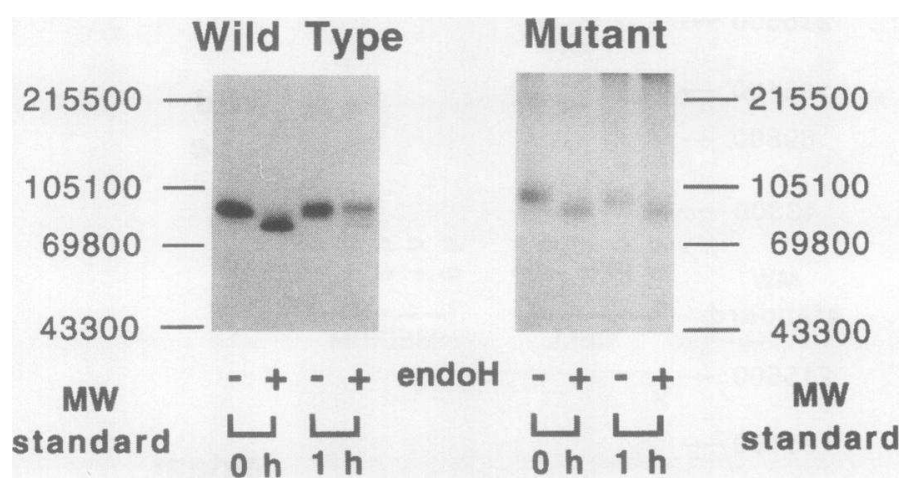

Figure 5. Endo $\mathrm{H}$ treatment of intracellular $b$ subunit. Cell lysates of the wild-type and mutant $b$ subunits pulse-labeled in BHK cells were treated with $(+)$ or without $(-)$ Endo $\mathrm{H}$ for $16 \mathrm{~h}$ at $37^{\circ} \mathrm{C}$ and were electrophoresed on $10 \%$ SDS-polyacrylamide gels, followed by autoradiography. wild-type and mutant $b$ subunit were transfected into BHK cells. Such a mammalian expression system allows elucidation of the structural as well as functional consequences of the mutation in its biosynthesis.

The present studies demonstrated that the Cys430-Phe mutation does not prevent the de novo synthesis of the $b$ subunit. Since fairly large amounts of the mutant $b$ subunit were detected inside the cells of stable expression, severe defects in its transcription and/or translation are unlikely to have dramatic effects. Extracellular degradation of the mutant molecule was also excluded since no evidence was found when degradation in culture media was monitored over time. Instead, it has been clearly shown that the Cys430-Phe substitution impairs the secretion of newly synthesized mutant $b$ subunit (Figs. 3 and 4).

Two general aberrant posttranslational patterns of biosynthesis resulting in reduced secretion from cells, intracellular accumulation and intracellular degradation of the newly synthesized protein, have been demonstrated in $\alpha_{1}$-antitrypsin deficiency (19-23). Both $\alpha_{1}$-antitrypsin and the $b$ subunit for factor XIII are typical secretary proteins produced by hepatocytes. Secretary proteins newly synthesized in the ER are attached to high-mannose type oligosaccharides, which are further modified to become complex type oligosaccharides in the Golgi apparatus (24). In contrast to the wild-type $b$ subunit, the mutant was present inside BHK cells and was not secreted from the cells. To evaluate the basis of this reduced secretion, the intracellular form of the newly synthesized $b$ subunit was examined. It is

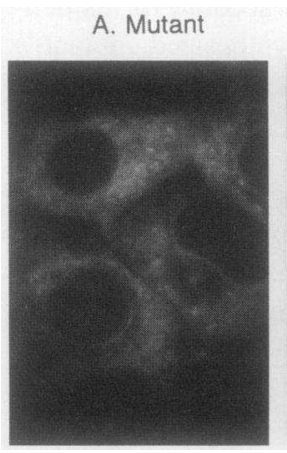

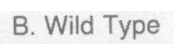

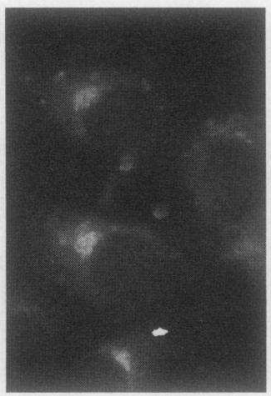

C. Mock

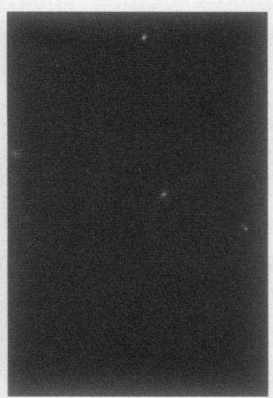

Figure 6. Localization of the $b$ subunit in BHK cells. Cells grown on coverslips were fixed, permealized, and subjected to indirect immunofluorescence using first a rabbit antiserum against the $b$ subunit and a goat anti-rabbit IgG antibody coupled to FITC in the second step. Displayed are cells producing the mutant $(A)$ and wild-type $(B)$, and control mock cells $(C)$. very likely that the mutant protein is trapped at some stage $(\mathrm{s})$ in the secretary pathway and has not undergone proper processing of oligosaccharides. When normal and mutant intracellular $b$ subunits were treated with endo $\mathrm{H}$, an enzyme which cleaves high-mannose but not complex type oligosaccharides, only the intracellular mutant remained sensitive to digestion (Fig. 5). Thus, it was concluded that the mutant $b$ subunit never reached the medial Golgi, and that the intracellular transport of the mutant $b$ subunit is impaired in BHK cells.

Impaired intracellular transport of mutant proteins is considered to be a general mechanism for various protein deficiencies (25-30). In most cases, the cause of impaired transport of mutants was attributed to ER retention because of the sensitivity to endo $\mathrm{H}$ treatment. Few trapped mutant proteins were actually shown to be localized inside the ER $(26,30)$. In the present study, the mutant $b$ subunit was shown by immunocytochemistry to remain exclusively inside the synthesizing cells (Fig. 6), very likely in the ER. However, the precise mechanisms for the ER retention per se are not known. The pronounced homology among Sushi domain-containing proteins permits an alignment of highly conserved amino acid residues $(7,9)$. In particular, four Cys residues in each Sushi domain are found in identical positions in every member of this protein superfamily. The first and third, and the second and fourth Cys residues are linked together, forming two disulfide bonds $(31,32)$. It is likely that the secondary and three-dimensional structures of Sushi domains are also similar (33-35). The loss of a Cys residue by the Cys430-Phe substitution disrupts the Cys430-Cys405 disulfide bond in the seventh Sushi domain of the $b$ subunit and leaves Cys405 unpaired. The resulting seventh Sushi domain may alter its gross conformation, likely becoming more extended than the normal molecule which is compacted by two disulfide bonds. The difference in electrophoretic mobility shown in Fig. 2 supports this possibility. However, the alteration in conformation of the mutant $b$ subunit did not abolish its dimer formation.

Structural changes of the mutant molecule may increase its interactions with resident ER proteins (36), resulting in markedly delayed transport through the secretory pathway as shown by the pulse-chase experiments. Alternatively, a small change in the structure of the mutant molecule can have a profound negative influence on its interaction with other proteins which are required for posttranslational transport through the secretary pathway. Consequently, an alteration in the primary structure of a protein can result in decreased transport to the exterior of the cell. The structural characteristics that mediate the specific interactions of the $b$ subunit with the multiple components of 
the transport mechanism are not yet known and the occurrence of a mutation within a Sushi domain can have unpredictable consequences.

Proteins that have been retained in the ER because of improper folding and/or aberrant posttranslational processing are eventually degraded $(37,38)$. Thus, the misfolded mutant $b$ subunit may become a target of degradation systems inside hepatocytes. In the case of the mutant $b$ subunit expressed in BHK cells, it is evident that the protein is degraded in these cells since intracellular accumulation of the newly synthesized mutant $b$ subunit molecule is not prominent.

In the case of $\alpha_{1}$-antitrypsin deficiency, intrahepatocyte defects in $\alpha_{1}$-antitrypsin biosynthesis associated with its mutations are manifest in like manners in all heterologous cells including fibroblasts, kidney cells, and monocytes (19-23). It is reasonable to assume that the abnormal intracellular transport of the mutant in BHK and COS-7 cells also occurs in hepatocytes and that the abnormal $b$ subunit never reaches circulation since the proband manifests complete deficiency. Direct evidence is not available, however, because liver biopsy of the proband has not been performed. Hepatoma cells were not employed for these experiments because they synthesize endogenous $b$ subunit (unpublished data), which hampers analyses.

Before the first case of complete $b$ subunit deficiency was found in Japan (4), several patients demonstrating similar laboratory findings had been reported in Italy (39-41). These patients were classified as type I factor XIII deficiency, which is characterized by the absence of both $a$ and $b$ subunits (39). They may have separate defects in both genes by chance, resulting in real combined deficiency of both subunits. Alternatively, reduced plasma $a$ subunit levels in these cases may be secondary to the $b$ subunit deficiency, in the same manner as the Japanese case (4). Amounts of the $a$ subunit in platelets were not described in the reports for these Italian cases (3941). If there is no $a$ subunit in platelets (or placenta), the $a$ subunit deficiency is independent from the $b$ subunit deficiency. If normal amounts of the $a$ subunit are present in platelets, the gene for the $a$ subunit must be normal and that for the $b$ subunit must have some defect(s). To examine both genes, we have been analyzing the DNAs obtained from two of these patients with type I factor XIII deficiency. (DNAs of two patients with type I factor XIII deficiency are being examined in collaboration with Drs. Rodeghiero, Castaman, and Girolami.) Preliminary DNA sequence analysis revealed that there are no apparent abnormalities in the gene for the $a$ subunit and that they do not have the same mutations in the gene for the $b$ subunit as did the Japanese case (Hashiguchi et al., unpublished data). Many more patients with $b$ subunit deficiency will be discovered and elucidation of their genetic abnormalities will contribute to our understanding of the structure-function relationship in the $b$ subunit molecule in the near future.

Note added in proof. Tossetto et al. reported that the $a$ subunit was present and functional in the platelets of Type I factor XIII deficiency (Tossetto, A., G. Castaman, and F. Redeghiero. 1993. Thromb. Haemost. 69:1295).

\section{Acknowledgments}

The authors thank Professor E. W. Davie and Dr. D. Foster for their support in the initial period of this study, Dr. M. Yamakawa and Profes- sor Y. Imai for their help in immunocytochemical experiments, Dr. F. Tokunaga for his helpful discussion, and Professor T. Yoshida for making tissue culture facilities available in the beginning. The authors are also grateful to Mr. H. Kaetsu for his help and discussion throughout the study, Dr. Tomonori Izumi for performing nondenatured polyacrylamide gel electrophoresis, and Ms. L. Boba for her help in preparation of the manuscript.

This work was supported in part by research grants from the Mochida Memorial Foundation, the Ichiro Kanehara Foundation, and Japan Research Foundation for Clinical Pharmacology.

\section{References}

1. Folk, J. E., and S. I. Chung. 1975. Blood coagulation factor XIII: relationship of some biological properties to subunit structure. In Proteases and Biological Control. E. Reich, D. B. Rifken, and E. Shaw, editors. Cold Spring Harbor Laboratory Press, Cold Spring Harbor, NY. 157-170.

2. Lorand, L., M. S. Losowsky, and K. J. M. Miloszewski. 1980. Human factor XIII: Fibrin-stabilizing factor. Prog. Thromb. Hemostas. 5:245-290.

3. Ichinose, A. 1994. The physiology and biochemistry of factor XIII. In Haemostasis and Thrombosis. A. L. Bloom, C. D. Forbes, D. P. Thomas, and E. G. D. Tuddenham, editors. Churchill Livingstone, Edinburgh. 531-546.

4. Saito, M., H. Asakura, T. Yoshida, K. Ito, K. Okafuji, T. Yoshida, and T. Matsuda. 1990. A familial factor XIII subunit B deficiency. Br. J. Haematol. 74:290-294.

5. Ichinose, A., L. E. Hendrickson, K. Fujikawa, and E. W. Davie. 1986. Amino acid sequence of the $a$ subunit of human factor XIII. Biochemistry. 25:6900-6906.

6. Ichinose, A., and E. W. Davie. 1988. Characterization of the gene for the $a$ subunit of human factor XIII (plasma transglutaminase), a blood coagulation factor. Proc. Natl. Acad. Sci. USA. 85:5829-5833.

7. Ichinose, A., B. A. McMullen, K. Fujikawa, and E. W. Davie. 1986. Amino acid sequence of the $b$ subunit of human factor XIII, a protein composed of ten repetitive segments. Biochemistry. 25:4633-4638.

8. Bottenus, R. E., A. Ichinose, and E. W. Davie. 1990. Nucleotide sequence of the gene for the $b$ subunit of human factor XIII. Biochemistry. 29:1119511209.

9. Ichinose, A., R. E. Bottenus, and E. W. Davie. 1990. Structure of transglutaminases. J. Biol. Chem. 265:13411-13414.

10. Kamura, T., T. Okamura, M. Murakawa, H. Tsuda, T. Teshima, T. Shibuya, M. Harada, and Y. Niho. 1992. Deficiency of coagulation factor XIII A subunit caused by the dinucleotide deletion at the $5^{\prime}$ end of exon III. J. Clin. Invest. 90:315-319.

11. Board, P., M. Coggan, and K. Miloszewski. 1992. Identification of a point mutation in factor XIII A subunit deficiency. Blood. 80:937-941.

12. Ichinose, A., and H. Kaetsu. 1993. Molecular approach to the structurefunction relationship of human coagulation factor XIII. Methods Enzymol. 222:36-51.

13. Standen, G. R., and D. J. Bowen. 1993. Factor XIII $A_{\text {Bristol 1 : detection of }}$ a nonsense mutation ( $\mathrm{Arg}^{171}$-stop codon) in factor XIII A subunit deficiency. Br. J. Haematol. 85:769-772.

14. Hashiguchi, T., M. Saito, E. Morishita, T. Matsuda, and A. Ichinose. 1993. Two genetic defects in a patient with complete deficiency of the $b$ subunit for factor XIII. Blood. 82:145-150.

15. Busby, S. J., E. Mulvihill, D. Rao, A. A. Kumar, P. Lioubin, M. Heipel, C. Sprecher, L. Halfpap, D. Prunkard, J. Gambee, and D. C. Foster. 1991. Expression of recombinant human plasminogen in mammalian cells is augmented by suppression of plasmin activity. J. Biol. Chem. 266:15286-15292.

16. Tayler, J. W., W. Schmidt, R. Cosstick, A. Okruszek, and F. Eckstein. 1985. The use of phosphorothioate-modified DNA in restriction enzyme reactions to prepare nicked DNA. Nucleic Acids Res. 13:8749-8785.

17. Lorand, L., J.-M. Jeong, J. T. Radek, and J. Wilson. 1993. Human plasma factor XIII: Subunit interactions and activation of zymogen. Methods Enzymol. 222:22-35.

18. Gross, V., T. Andus, T.-A. Tran-Thi, R. T. Schwarz, K. Decker, and P. C. Heinrich. 1983. 1-Deoxynojirimycin impairs oligosaccharide processing of $\alpha_{1}$ proteinase inhibitor and inhibits its secretion in primary cultures of rat hapatocytes. J. Biol. Chem. 258:12203-12209.

19. Verbanac, K. M., and E. C. Heath. 1986. Biosynthesis, processing, and secretion of $\mathrm{M}$ and $\mathrm{Z}$ variant human $\mathrm{a}_{1}$-antitrypsin. J. Biol. Chem. 261:99799989.

20. Curiel, D. T., M. D. Holmes, H. Okayama, M. L. Brantly, C. Vogelmeier, W. D. Travis, L. E. Stier, W. H. Perks, and R. G. Crystal. 1989. Molecular basis of the liver and lung disease associated with the $a_{1}$-antitrypsin deficiency allele

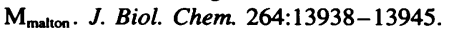

21. Brantly, M., M. Courtney, and R. G. Crystal. 1988. Repair of the secretion defect in the $\mathrm{Z}$ form of $\boldsymbol{\alpha}_{1}$-antitrypsin deficiency by addition of a second mutation. Science (Wash. DC). 242:1700-1702. 
22. Curiel, D. T., A. Chytil, M. Courtney, and R. G. Crystal. 1989. Serum $\alpha_{1^{-}}$ antitrypsin deficiency associated with the common S-type (Glu264-Val) mutation results from intracellular degradation of $\alpha_{1}$-antitrypsin prior to secretion. J. Biol. Chem. 264:10477-10486.

23. Curiel, D. T., C. Vogelmeier, R. C. Hubbard, L. E. Stier, and R. G. Crystal. 1990. Molecular basis of $\alpha_{1}$-antitrypsin deficiency and emphysema associated with the $\alpha_{1}$-antitrypsin $M_{\text {mineral springs }}$ allele. Mol. Cell. Biol. 10:47-56.

24. Kornfeld, R., and S. Kornfeld. 1985. Assembly of asparagine-linked oligosaccharide. Ann. Rev. Biochem. 54:631-664.

25. Miura, O., Y. Sugahara, and N. Aoki. 1989. Hereditary $\alpha_{2}$-plasmin inhibitor deficiency caused by a transport-deficient mutation ( $\alpha_{2}$ PI-Okinawa $)$. J. Biol. Chem. 264:18213-18219.

26. Tsuji, E., Y. Misumi, T. Fujiwara, N. Takami, S. Ogata, and Y. Ikehara 1992. An active-site mutation (Gly ${ }^{633}$-Arg) of dipeptidyl peptidase IV causes its retention and rapid degradation in the endoplasmic reticulum. Biochemistry. 31:11921-11927.

27. Lyons, S. E., M. E. Bruck, E. J. W. Bowie, and D. Ginsburg. 1992. Impaired intracellular transport produced by a subset of type IIA von Willebrand disease mutations. J. Biol. Chem. 267:4424-4430.

28. Poncz, M., S. Rifat, B. S. Coller, P. J. Newman, S. J. Shattil, and T. Parrella, P. Fortina, and J. S. Bennett. 1994. Glanzmann Thromboasthenia secondary to a Gly273-Asp mutation adjacent to the first calcium-binding domain of platelet glycoprotein IIb. J. Clin. Invest. 93:172-179.

29. Tolleshaug, H., K. K. Hobgood, M. S. Brown, and J. L. Goldstein. 1983. The LDL receptor locus in familial hypercholesterolemia: Multiple mutations disrupt transport and processing of a membrane receptor. Cell. 32:941-951.

30. Cheng, S. H., R. J. Gregory, J. Marshall, S. Paul, D. W. Souza, G. A. White, C. R. O'Riordan, and A. E. Smith. 1990. Defective intracellular transport and processing of CFTR is the molecular basis of most cystic fibrosis. Cell. 63:827-834
31. Janatova, J., K. G. M. Reid, and A. C. Willis. 1989. Disulfide bonds are localized within the short consensus repeat units of complement regulatory proteins: C4b-binding protein. Biochemistry. 28:4754-4761.

32. Kato, H., and K. Enjoji. 1991. Amino acid sequence and location of the disulfide bonds in bovine $\beta_{2}$-glycoprotein I: The presence of five sushi domains. Biochemistry. 30:11687-11694.

33. Perkins, S. J., P. I. Haris, R. B. Sim, and D. Chapman. 1988. A study of the structure of human complement component factor $\mathrm{H}$ by Fourier transform infrared spectroscopy and secondary structure averaging methods. Biochemistry. 27:4004-4012.

34. Carrell, N. A., H. P. Erickson, and J. McDonagh. 1989. Electron microscopy and hydrodynamic properties of factor XIII subunits. J. Biol. Chem 264:551-556.

35. Dahlback, B., C. A. Smith, and H. J. Muller-Eberhard. 1983. Visualization of human $\mathrm{C} 4 \mathrm{~b}$-binding protein and its complexes with vitamin $\mathrm{K}$-dependent protein S and complement protein C4b. Proc. Natl. Acad. Sci. USA. 80:3461-3465.

36. Klausner, R. D., and R. Sitia. 1990. Protein degradation in the endoplasmic reticulum. Cell. 62:611-614.

37. Hurtley, S. M., and A. Helenius. 1989. Protein oligomerization in the endoplasmic reticulum. Annu. Rev. Cell Biol. 5:277-307.

38. Pelman, H. R. B. 1989. Control of protein exit from the endoplasmic reticulum. Annu. Rev. Cell Biol. 5:1-23.

39. Girolami, A., A. Burul, F. Fabris, G. Cppellato, and C. Betterle. 1978 Studies on factor XIII antigen in congenital factor XIII deficiency. A tentative classification of the disease in two groups. Folia Haematol. 105:131-141.

40. Cappellato, M. G., A. R. Lazzaro, F. Marafioti, G. Polato, and A. Girolami. 1987. A new family with congenital factor XIII deficiency showing a deficit of both subunit a and b. Type I factor XIII deficiency. Haematologia. 20:179-187.

41. Rodeghiero, F., A. Tosetto, E. Di Bona, and G. Castaman. 1991. Clinica pharmacokinetics of a placenta-derived factor XIII concentrate in type I and type II factor XIII deficiency. Am. J. Hematol. 36:30-34. 